\title{
O PAPEL DO OURO NA COBERTURA DO RISCO DA TAXA DE CÂMBIO EURO-DÓLAR
}

GOLD AS A HEDGE AGAINST THE EURO-DOLLAR EXCHANGE RATE EL PAPEL DEL ORO EN LA COBERTURA DEL RIESGO DE CAMBIO EURO-DÓLAR

Júlio Lobão (jlobao@fep.up.pt)*

Juliana Teixeira

\section{RESUMO:}

Neste artigo é investigado o papel desempenhado pelo ouro na cobertura do risco da taxa de câmbio euro-dólar no período 1999-2011. Para isso, recorreu-se a um modelo EGARCH $(2,1,1)$ aplicado a dados diários. Os resultados mostram que o ouro se revelou útil para a cobertura do risco cambial dos investimentos em dólares levados a cabo pelos habitantes da zona-euro. Durante todo o período considerado, a cotação do ouro mostrouse inelástica em relação à taxa de câmbio euro-dólar tanto no curto como no longo prazo.

Palavras-chave: cobertura de risco, ouro, taxa de câmbio.

\section{ABSTRACT:}

In this article we investigate the role of gold as hedge of the euro-dollar exchange rate during the period 1999-2011. For this, it was used an EGARCH $(2,1,1)$ model applied to daily data. The results show that gold has served as an useful hedge against the exchange rate risk of the investments in dollardenominated assets carried out by the inhabitants of the euro-zone. During the period of the sample, gold was inelastic with respect to the euro-dollar exchange rate both in the short-run and in the long-run.

Keywords: exchange rate, gold, hedge.

\section{RESUMEN:}

En este trabajo se investigó el papel del oro en la cobertura del riesgo de tasa de cambio euro-dólar en el período 1999-2011. Para ello, se utilizó un modelo EGARCH $(2,1,1)$ aplicado a datos diarios. Los resultados muestran que el oro ha demostrado ser útil para la cobertura del riesgo de cambio de las inversiones en dólares realizadas por los habitantes de la zona euro. A lo largo del período considerado, el precio del oro se ha mostrado mostrado inelástica en relación al cambio euro-dólar en el corto y largo plazo.

Palabras-clave: cobertura del riesgo, oro, tasa de cambio. 
* Júlio Lobão. Professor Auxiliar na Faculdade de Economia, Universidade Porto. Mestre em Finanças 


\section{INTRODUÇÃO}

Apesar dos metais preciosos terem sido usados como meio de troca e de reserva de valor durante milhares de anos, o interesse acerca do ouro enquanto ativo financeiro é relativamente recente. Esse interesse foi grandemente aumentado pela atual crise financeira. A maior volatilidade do mercado acionista e os receios associados à dívida soberana fizeram com que os investidores encarassem o metal amarelo como mais um ativo financeiro, suscetível de ser incluído nas carteiras de investimento.

aumento na procura de ouro repercutiu-se numa fortíssima subida do seu preço, ao mesmo tempo que os instrumentos tradicionais de investimento registavam perdas. No período de três anos que se seguiu à falência do banco Lehman Brothers, o preço de mercado do ouro foi multiplicado por um fator de seis, passando de pouco menos de 300 dólares a onça em Setembro de 2008 para quase 1800 dólares a onça nos finais do ano de 2011.

$\bigcirc$ grande interesse nos investimentos em ouro trouxe o metal para a ordem do dia tendo passado a ser referido frequentemente nos meios de comunicação social. No entanto, apesar da sua popularidade, uma pesquisa na literatura acerca do papel do ouro enquanto investimento financeiro revela um escasso número de estudos quando comparado com a investigação realizada acerca do mercado acionista ou obrigacionista, por exemplo.

$\bigcirc$ presente artigo pretende contribuir para o estudo do ouro enquanto ativo financeiro. Mais concretamente, o objetivo é o de investigar empiricamente os resultados do investimento em ouro com o propósito da cobertura do risco da taxa de câmbio do euro contra o dólar. Para isso, recorre-se à aplicação de um modelo EGARCH $(2,1,1)$ a dados de frequência diária referentes ao período que vai desde a introdução da moeda europeia (em 1999) até ao ano de 2011. Os resultados sugerem que, nesse período, o ouro se mostrou como um ativo capaz de cobrir o risco cambial inerente ao investimento em ativos denominados em dólares por parte dos agentes residentes na zonaeuro.

Esta contribuição afigura-se relevante uma vez que, tanto quanto é do nosso conhecimento, trata-se do primeiro estudo em que se testa a utilidade do ouro para a cobertura do risco da taxa de câmbio euro-dólar.

artigo está organizado da seguinte forma: na secção seguinte apresenta-se a revisão da literatura relevante. A terceira secção é dedicada à análise dos dados e dos resultados do estudo empírico. Na 
secção final resume-se o artigo e referem-se sugestões para investigação futura.

\section{REVISÃO DA LITERATURA}

Diversos autores têm estudado o ouro enquanto ativo financeiro capaz de contribuir para a cobertura do risco dos investimentos. Um primeiro conjunto de estudos debruça-se sobre o papel do ouro na cobertura do risco de inflação. Ghosh, Levin, MacMillan e Wright (2004) aplicam testes de cointegração a dados mensais de preços do ouro entre 1976 e 1999. Como sublinham os autores, apesar da volatilidade do ouro no curto prazo poder levantar dificuldades à cobertura do risco de inflação, a relação estável entre o preço do metal e a inflação em prazos longos torna o ouro um ativo eficaz na cobertura desse risco. Worthington e Pahlavani (2007), através da análise às relações de cointegração do metal precioso com a inflação nos EUA entre os anos de 1946 e de 2006, corroboram a conclusão de Ghosh et al. (2004). Estes resultados são também confirmados por McCown e Zimmerman (2006), com um método semelhante, para uma amostra mais limitada (de 1970 a 2003).

Um segundo grupo de autores investiga a relação entre os preços do ouro e das ações. As conclusões apontam para a utilidade do ouro na cobertura do risco de variação do preço desses ativos. Para Sherman (1983), por exemplo, um investimento em ouro numa proporção de 5\% a 10\% da carteira é suficiente para diminuir a volatilidade e aumentar a rendibilidade obtida. Jaffe (1989) mostra que a correlação entre o ouro (representado pelos derivados do metal) e as ações é muito baixa. Por isso, embora o metal comporte um risco assinalável enquanto investimento isolado, pode desempenhar um papel importante na diversificação das carteiras de ações. Para Chua, Stick e Woodward (1990), o peso dos investimentos em ouro deve ser maior. $\bigcirc$ autor conclui que é necessário um investimento em ouro equivalente a 25\% da carteira para se reduzir o risco (e aumentar a rendibilidade) com significado. Lucey, Tully e Poti (2006) mostram que o preço do ouro apresentou uma correlação negativa com os índices acionistas NYSE, NASDAQ e FTE no período 1988-2003. Os autores defendem que a diversificação pela detenção de ouro é particularmente útil em alturas de crise (caracterizadas por instabilidade política, colapso dos mercados de ações ou fraudes corporativas, por exemplo). Outros autores chegam a conclusões semelhantes. Por exemplo, Baur e McDermott (2010) utilizam uma amostra de 30 anos (1979-2009) relativa aos índices acionistas de 13 países desenvolvidos e 
emergentes para concluir que o investimento em ouro não só permite cobrir o risco de variação dos preços das ações na generalidade dos países desenvolvidos, como se revela um ativo de refúgio particularmente útil em períodos de crise. Draper, Faff e Hillier (2006) e Faugere e Erlach (2005) são outros autores que realçam a utilidade do ouro na cobertura do risco inerente ao investimento em ações.

Uma terceira óptica de análise diz respeito à utilização do ouro para cobertura do risco cambial. Capie, Mills e Wood (2005) utilizam um modelo EGARCH para concluir que o investimento em ouro permite cobrir o risco de depreciação do dólar face à libra esterlina e ao iene embora essa capacidade de cobertura do risco tenha variado ao longo do tempo com o papel desempenhado pelo ouro. A principal distinção é estabelecida entre o período em que o ouro esteve na base da emissão monetária (ou seja, até o ano de 1971) e o período que se the seguiu.

\section{DADOS E ESTUDO EMPÍRICO}

A amostra utilizada é constituída pelos dados com frequência diária do preço do ouro e da taxa de câmbio euro-dólar. Ambas as séries estatísticas foram retiradas da base de dados Datastream. 0 período da amostra está compreendido entre o dia 4 de Janeiro de 1999 (data de introdução do euro como moeda escritural) e o dia 24 de Outubro de 2011 totalizando 3341 observações.

A taxa de câmbio euro-dólar apresenta-se com a forma de cotação ao certo para o euro, ou seja, exprime o número de dólares por cada euro. O preço do ouro encontra-se cotado em dólares por onça troy.

As duas séries encontram-se representadas no gráfico seguinte: 


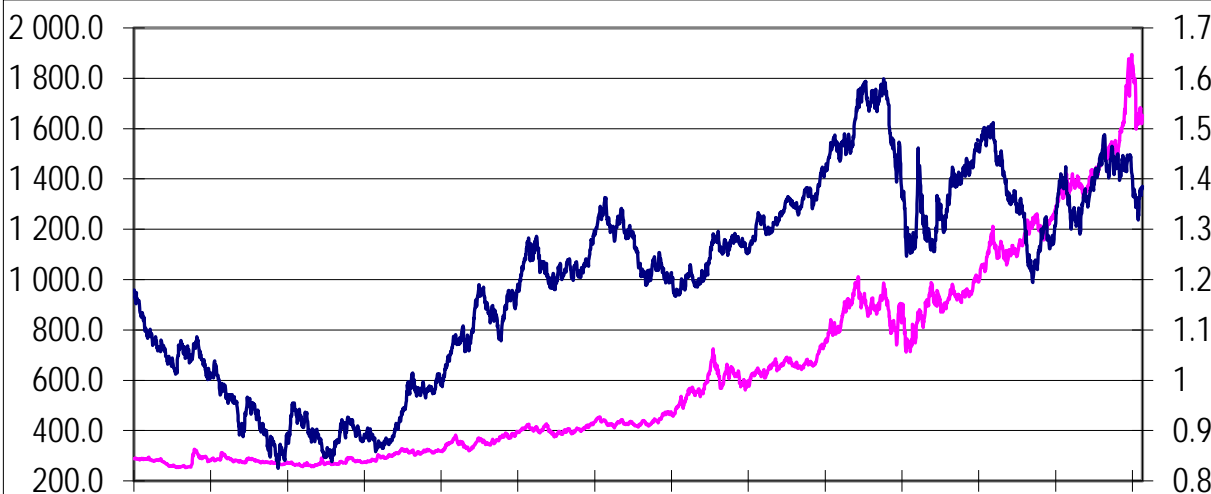

01- 12- 12- 12- 11- 11- 11- 10- 10- 10- 10- 09- 09- 0919991999200020012002200320042005200620072008200920102011

ouro (escala da esquerda)

Figura 1 - Evolução do preço do ouro e da taxa de câmbio euro-dólar entre 1/01/1999 e 24/10/2011; Fonte dos dados: DataStream

Como se pode constatar, registou-se uma subida muito significativa no preço do ouro durante o período da amostra. Na primeira sessão de 1999 o ouro encontrava-se cotado a 287,2 dólares por onça enquanto que o preço no dia 24 de Outubro de 2011 era de 1652 dólares o que representa um incremento de cerca de 475\% em pouco menos de 13 anos. $O$ aumento dos preços foi particularmente acentuado no período entre Novembro de 2008 e Setembro de 2011. Nesses 22 meses, o preço do ouro passou dos 725 dólares para um máximo histórico de quase 1900 dólares (aumento de cerca de 160\%). Deve também ser assinalada a correção dos preços em relação a esse máximo: a cotação desceu quase 300 dólares (cerca de 14\%) em cerca de um mês.

A evolução da taxa de câmbio euro-dólar está também representada na figura 1. $\bigcirc$ comportamento tem sido, na globalidade do período, de apreciação do euro face ao dólar mas com importantes períodos de depreciação do euro (apreciação do dólar) como nos primeiros seis meses após a introdução da moeda única, no final do ano de 2008 e na primeira metade do ano de 2010. O valor máximo do euro contra a moeda dos EUA verificou-se em Abril de 2008 e o valor mínimo no mês de Outubro de 2010. Comparando os valores iniciais e final da taxa de câmbio verificamos que a apreciação do euro se situou nos $17,53 \%$.

Dada a elevada variação de cada uma das séries, utilizaram-se as rendibilidades logarítmicas para o preço do ouro e para a cotação euro-dólar. Assim, a análise econométrica foi levada a cabo com a 
primeira diferença nos logaritmos do preço do ouro, adiante representada por $\Delta g_{t}$, e pela primeira diferença nos logaritmos da taxa de câmbio, adiante designada por $\Delta s_{t}$

Seguindo a prática corrente na literatura, como por exemplo em Capie et al. (2005) e em Baur e McDermott (2010), pressupomos que os erros de previsão são, no caso de preços como a cotação do ouro ou taxas de câmbio, altamente variáveis em diferentes períodos de tempo. A variação dos erros de previsão de variáveis de mercado aconselha a utilização de modelos heterocedásticos do tipo Autoregressive Conditional Heterocedasticity (ARCH). Para determinar a variante do modelo ARCH mais adequada para o caso consideramos o modelo ARCH generalizado (GARCH) proposto por Bollerslev (1986), o modelo GARCH exponencial (EGARCH) desenvolvido por Nelson (1991) e o modelo GJR criado por Glosten, Jagannathan e Runkle (1993). Entre os modelos GARCH, EGARCH e GJR e as distribuições normal e t-student foram projetados 47 hipóteses de modelos considerando no máximo três desfasamentos (ver anexo). Seguidamente, das 47 hipóteses de modelos foram selecionados quatro modelos de acordo com os critérios de informação de Akaike e de Schwarz. ${ }^{18}$ Este procedimento conduziu à escolha dos modelos EGARCH $(1,1,1)$, EGARCH $(2,1,1)$, EGARCH $(1,2,2)$ e EGARCH $(3,3,3)$, todos com uma distribuição t-student. Os dois primeiros são os que apresentam um menor valor no critério de Schwarz e os dois últimos apresentam os menores valores no critério de informação de Akaike. ${ }^{19}$

$\bigcirc$ teste de Engle à heterocedasticidade residual foi utilizado como critério de desempate entre os quatro modelos. ${ }^{20} \bigcirc$ teste conduziu à selecção do modelo EGARCH $(2,1,1)$ uma vez que é o único em que não se rejeita, a 10\% de significância estatística, a hipótese de inexistência de heterocedasticidade condicional (efeitos ARCH). Como termo de comparação refira-se que Capie et al. (2005)

\footnotetext{
${ }^{18}$ Os critérios de Akaike e de Schwarz medem a qualidade relativa do modelo estatístico, estando definida a amostra de dados. Permitem seleccionar um modelo entre diversas alternativas tendo em atenção a complexidade do modelo, nomeadamente o número de parâmetros do mesmo, e a qualidade de ajustamento aos dados da amostra.

19 Para averiguar das qualidades estatísticas dos quatro modelos foram ainda efectuados testes de autocorrelação dos resíduos e de autocorrelação dos resíduos quadrados. Em ambos os testes os resultados sugerem a ausência de efeitos $\mathrm{ARCH}$ em todos os modelos.

${ }^{20} \bigcirc$ teste de Engle à heterocedasticidade residual tem como objetivo aferir se os modelos evidenciam efeitos ARCH. Para isso, testa-se a hipótese nula de que a série de resíduos $\boldsymbol{r}_{\boldsymbol{t}}$ não apresentam heterocedasticidade condicional (efeitos $\mathrm{ARCH}$ ) contra a hipótese alternativa de que um modelo ARCH (L) representado por $r_{t}^{2}=\alpha_{0}+\alpha_{1} r_{t-1}^{2}+\ldots+\alpha_{L} r_{t-L}^{2}+e_{t}$ descreva a série com pelo menos um $\alpha_{k}$ não nulo para $k=0, \ldots, L$. Acerca deste teste, ver Engle (1988).
} 
seleccionaram um modelo EGARCH $(1,2,1)$ enquanto que Baur e McDermott (2010) utilizaram um modelo GARCH $(1,1)$.

Para um modelo EGARCH, as expressões da média e da variância são, respectivamente, as seguintes:

$$
\begin{aligned}
& \Delta g_{t}=\beta_{0}+\beta_{1} \Delta s_{t}+\beta_{2} \Delta g_{t-1}+\mu_{t} \\
& \log \left(\sigma_{t}^{2}\right)=\alpha_{0}+\alpha_{1} \frac{\mu_{t-1}}{\sigma_{t-1}}+\alpha_{2} \log \left(\sigma_{t-1}^{2}\right)+\alpha_{3} \frac{\left|\mu_{t-1}\right|}{\sigma_{t-1}}+\alpha_{4} \log \left(\sigma_{t-2}^{2}\right)
\end{aligned}
$$

A estimativa dos coeficientes do modelo $\operatorname{EGARCH}(2,1,1) \mathrm{com}$ os dados da amostra referida conduziu aos seguintes resultados:

$$
\Delta g_{t}=\underset{(0,000143)}{0,000636}+\underset{(0,020512)}{0,276962} \Delta s_{t}-\underset{(0,016810)}{0,082719} \Delta g_{t-1}
$$

$$
\begin{gathered}
\log \left(\sigma_{t}^{2}\right)=\underset{(0,027873)}{-0,151787}+\underset{(0,009717)}{0,062970} \frac{\mu_{t-1}}{\sigma_{t-1}}+\underset{(0,002513)}{0,991293} \log \left(\sigma_{t-1}^{2}\right)+\underset{(0,038304)}{0,147059} \frac{\left|\mu_{t-1}\right|}{\sigma_{t-1}} \\
-\underset{(0,039625)}{0,047941} \frac{\left|\mu_{t-2}\right|}{\sigma_{t-2}}
\end{gathered}
$$

Todas as estimativas se mostraram significativas a 1\% de significância com exceção de $\alpha_{4}$ (p-value de 0,2263).

Relativamente à variação da volatilidade ao longo do tempo, temos que o modelo será estacionário em média se $0<\left|\beta_{2}\right|<1$ e será estacionário em variância se $\alpha_{2}<1$. No caso, o modelo EGARCH $(2,1,1)$ com distribuição t-student estimado revela-se estacionário em ambas as situações uma vez que $\beta_{2}=-0,082719$ e que $\alpha_{2}=0,991293$

Os resultados obtidos permitem extrair diversas conclusões. Em primeiro lugar, verifica-se que a estimativa de $\beta_{1}$ é positiva o que nos leva a concluir que o ouro permitiu a cobertura do risco cambial dos investimentos em dólares levados a cabo pelo habitantes da zona-euro. De facto, a variação da taxa de câmbio euro-dólar dá-se no mesmo sentido do preço do ouro. Isso significa, por exemplo, que um aumento dessa taxa de câmbio, o que implica a uma depreciação dos investimentos em dólares quando convertidos em euros, é compensada pela variação favorável (ou seja, positiva) da cotação do ouro. 
Em segundo lugar, como $\beta_{1}$ corresponde à elasticidade de curto prazo, pode-se afirmar que a cotação do ouro se tem mostrado inelástica no período em análise relativamente à taxa de câmbio eurodólar. Relativamente à estimativa da elasticidade de longo prazo temos

que:

prazo (5)

Verifica-se que a estimativa do efeito de curto prazo é ligeiramente superior à estimativa de efeito de longo prazo indicando que a resposta instantânea da cotação do ouro a uma variação da taxa de câmbio euro-dólar é superior à variação verificada no metal precioso a longo prazo.

Em terceiro lugar, temos os resultados da análise aos efeitos de assimetria na volatilidade face a variações não esperadas do preço do ouro. Nessa análise não foi considerado o valor de $\alpha_{4}$ por não ser, como se referiu acima, estatisticamente significativo. Ora, quando $\mu_{t-1}>0$, a volatilidade do preço do ouro é dada por $\alpha_{1}+\alpha_{3}=0,210029$. Já quando $\mu_{t-1}<0$, essa volatilidade é dada por $-\alpha_{1}+\alpha_{3}=0,084089$. A existência de uma assimetria positiva leva-nos a concluir, portanto, que uma subida do preço do ouro superior à esperada ( $\left.\mu_{t-1}>0\right)$, é acompanhada de maior volatilidade do que uma descida do ouro superior à esperada $\left(\mu_{t-1}<0\right)$.

Por último, podemos observar que, pelo facto de $\alpha_{2}=0,991293$, ou seja, muito próximo da unidade, os efeitos dos choques não antecipados na taxa de variação do preço do ouro são bastantes persistentes, implicando a manutenção de uma volatilidade acrescida durante um período de tempo considerável.

Dado o comportamento do preço do ouro ser muito diferenciado durante o período da amostra, foi analisada a dispersão dos logaritmos do preço do ouro contra o logaritmo da taxa de câmbio euro/dólar. Tal como fizeram Capie et al. (2005), procedemos à identificação de diferentes estratos correspondentes aos diversos subperíodos da amostra. A primeira série temporal representa o período inicial do euro que vai desde 4 de Janeiro de 1999 até 30 de Dezembro de 2005. A segunda série coincide com a fase durante a qual o ouro registou o primeiro período de aumento significativo no seu preço, ou seja, desde 2 de Janeiro de 2006, até 29 de Agosto de 2008. A terceira e última série diz respeito à crise financeira, 
abrangendo o período desde 1 de Setembro de 2008 até ao final da amostra.

Foi realizada uma análise para cada um destes subperíodos tendo uma vez mais sido testados os vários modelos referidos em anexo. Considerando o critério de informação de Akaike foram selecionados os melhores modelos. A tabela 1 reúne os resultados obtidos para cada um dos subperíodos e para toda a amostra.

\begin{tabular}{|c|c|c|c|c|}
\hline \multicolumn{2}{|c|}{ Tabela 1 - Resumo dos resultados obtidos na amostra total e nos três sub-períodos sob análise } \\
\hline \begin{tabular}{c} 
Período da amostra \\
\hline $\begin{array}{c}04 / 01 / 1999- \\
24 / 10 / 2011\end{array}$
\end{tabular} & $\begin{array}{c}\text { Modelo } \\
\text { do erribuição }\end{array}$ & $\begin{array}{c}\text { Elasticidade } \\
\text { de curto } \\
\text { prazo }\end{array}$ & $\begin{array}{c}\text { Elasticidade de } \\
\text { longo prazo }\end{array}$ \\
\hline $\begin{array}{c}04 / 01 / 1999- \\
30 / 12 / 2005\end{array}$ & EGARCH $(1,1,1)$ & $\dagger(4,3056)$ & 0,2417 & 0,2220 \\
\hline $\begin{array}{c}02 / 01 / 2006- \\
29 / 08 / 2008\end{array}$ & EGARCH $(1,1,2)$ & $\dagger(10,2629)$ & 0,8678 & 0,7536 \\
\hline $\begin{array}{c}01 / 09 / 2008- \\
24 / 10 / 2011\end{array}$ & EGARCH $(1,3,1)$ & $\dagger(5,9536)$ & 0,1912 & 0,1787 \\
\hline
\end{tabular}

As estimativas das elasticidades do ouro face a variações da taxa de câmbio em cada um dos subperíodos bem como as características dos processos de variância condicional e a distribuição dos erros são indicadas na tabela 1. As estimativas das elasticidades da amostra completa, mencionadas na primeira linha da tabela, são efetivamente médias ponderadas das estimativas relativas aos subperíodos. Com exceção do período compreendido entre Janeiro de 2006 e o final de Agosto de 2008, a reação do preço do ouro à taxa de câmbio é rápida. E com exceção do subperíodo referido, as elasticidades de curto prazo e de longo prazo apresentam valores próximos. As estimativas dos efeitos de longo prazo são ligeiramente superiores às do curto prazo o que indica que a reação do preço do ouro a variações da taxa de câmbio euro-dólar se atenua (ainda que ligeiramente) a longo prazo.

\section{CONCLUSÕES E SUGESTÕES DE INVESTIGAÇÃO FUTURA}

Este artigo analisa o papel do ouro na cobertura do risco da taxa de câmbio euro-dólar. Através da aplicação de um modelo EGARCH $(2,1,1)$ foi possível concluir que o metal precioso permitiu cobrir o risco de variação dessa taxa de câmbio no período entre 1999 e 2011. Um investidor europeu que tivesse investido em ativos denominados em 
dólares teria beneficiado com a detenção de ouro uma vez que tal teria permitido a cobertura do risco de depreciação do dólar à moeda europeia. Este estudo sugere, assim, que o ouro pode desempenhar um papel importante na constituição das carteiras de investimento.

Os resultados do presente estudo corroboram os obtidos por Capie et al. (2005) que concluíram pela existência de efeitos benéficos do ouro na cobertura do risco cambial inerentes a investimentos denominados em libras e em ienes levados a cabo pelos investidores dos EUA:

Ao longo das últimas décadas algumas commodities têm vindo a adquirir os atributos dos ativos financeiros. A investigação do papel de outras commodities na gestão de carteiras, e mais especificamente na gestão do risco cambial, encontra-se ainda no seu início. Assim, sugere-se que estudos semelhantes incidindo sobre commodities como a prata ou a platina sejam desenvolvidos para aferir do seu papel na gestão de carteiras. 


\section{BIBLIOGRAFIA}

Baur, D.; McDermott, T. (2010) Is Gold a Safe Haven? International Evidence, Journal of Banking \& Finance, 34, 1886-1898.

Bollerslev, T. (1986) Generalized Autoregressive Conditional Heteroskedasticity, Journal of Econometrics, 31, 307-327.

Capie, F.; Mills, T. C.; Wood, G. (2005) Gold as a Hedge against the Dollar, Journal of International Financial Markets, Institutions and Money, 15, 343-352.

Chua, J.; Stick, G.; Woodward, R. (1990) Diversifying with Gold Stocks, Financial Analysts Journal, 46, 76-79.

Draper, P.; Faff, R.; Hillier, D. (2006) Do Precious Metals Shine? An Investment Perspective, Financial Analysts Journal, 62, 98-106.

Engle, R. (1988) Autoregressive Conditional Heteroskedasticity with Estimates of the Variance of United Kingdom Inflation." Econometrica, 96, 893-920.

Faugere, C.; Erlach, J. (2005) The Price of Gold: a Global Required Yield Theory, Journal of Investing, 14, 99-111.

Ghosh, D.; Levin, E.J.; MacMillan, D.; Wright, R.E. (2004) Gold as an Inflation Hedge?, Studies in Economics and Finance, 22, 1-25.

Glosten, R.; Jagannathan, R.; Runkle, D. (1993) On the Relation Between Expected Value and Volatility of the Nominal Excess Returns on Stocks, Journal of Finance, 48, 1779-1801. Jaffe, J. (1989) Gold and Gold Stocks as Investments for Institutional Portfolios, Financial Analysts Journal, 45, 53-59.

Lucey, B.; Tully, E.; Poti, V. (2006) International Portfolio Formation, Skewness and the Role of Gold, Frontiers in Finance and Economics, 3, 49-68.

McCown, J. R.; Zimmerman, J.R. (2006) Is Gold a Zero-beta Asset? Analysis of the Investment Potential of Precious Metals, Working paper, Social Science Research Network, acessível em http://papers.ssrn.com/sol3/papers.cfm?abstract_id=920496

Nelson, D.B. (1991) Conditional Heteroskedasticity in Asset Returns: A New Approach, Econometrica, 59, 347-370.

Sherman, E. (1983) A Gold Pricing Model, The Journal of Portfolio Management, Spring, 6870.

Worthington, A.C.; Pahlavani, M. (2007) Gold Investment as an Inflationary Hedge: Cointegration Evidence with Allowance for Endogenous Structural Breaks, Applied Financial Economics Letters, 3, 259-262. 


\section{Anexo}

Tabela 2 - Modelos heterocedásticos considerados para o estudo empírico

\begin{tabular}{|c|c|c|c|c|c|}
\hline Modelo & Distribuição & R2 & $\begin{array}{c}\text { Estatística } \\
\text { Durbin- } \\
\text { Watson }\end{array}$ & $\begin{array}{l}\text { Critério de } \\
\text { Akaike }\end{array}$ & $\begin{array}{l}\text { Critério de } \\
\text { Schwarz }\end{array}$ \\
\hline $\operatorname{GARCH}(1,1)$ & Normal & 0,035143 & 1,974066 & $-6,306027$ & $-6,295041$ \\
\hline $\operatorname{GARCH}(1,1)$ & T-student & 0,033486 & 1,933208 & $-6,418176$ & $-6,405359$ \\
\hline $\operatorname{GARCH}(1,2)$ & Normal & 0,035204 & 1,974181 & $-6,307567$ & $-6,294750$ \\
\hline $\operatorname{GARCH}(1,2)$ & T-student & 0,033478 & 1,933614 & $-6,417604$ & $-6,402957$ \\
\hline $\operatorname{GARCH}(2,1)$ & Normal & 0,035127 & 1,966609 & $-6,309657$ & $-6,296841$ \\
\hline $\operatorname{GARCH}(2,1)$ & T-student & 0,033483 & 1,933388 & $-6,417588$ & $-6,402941$ \\
\hline $\operatorname{GARCH}(2,2)$ & Normal & 0,034949 & 1,965081 & $-6,313009$ & $-6,298361$ \\
\hline $\operatorname{GARCH}(2,2)$ & T-student & 0,033493 & 1,933246 & $-6,416983$ & $-6,400505$ \\
\hline $\operatorname{EGARCH}(1,1,1)$ & Normal & 0,034681 & 1,964180 & $-6,315812$ & $-6,302996$ \\
\hline $\operatorname{EGARCH}(1,1,1)$ & T-student & 0,032956 & 1,929354 & $-6,429362$ & $-6,414715$ \\
\hline $\operatorname{EGARCH}(1,1,2)$ & Normal & 0,034681 & 1,964180 & $-6,315812$ & $-6,302996$ \\
\hline $\operatorname{EGARCH}(1,1,2)$ & T-student & 0,034192 & 1,938378 & $-6,416466$ & $-6,399988$ \\
\hline $\operatorname{EGARCH}(1,2,1)$ & Normal & 0,034680 & 1,963466 & $-6,317124$ & $-6,302477$ \\
\hline $\operatorname{EGARCH}(1,2,1)$ & T-student & 0,032963 & 1,928658 & $-6,428799$ & $-6,412321$ \\
\hline $\operatorname{EGARCH}(2,1,1)$ & Normal & 0,034864 & 1,966846 & $-6,321790$ & $-6,307142$ \\
\hline $\operatorname{EGARCH}(2,1,1)$ & T-student & 0,033134 & 1,930143 & $-6,429143$ & $-6,412665$ \\
\hline $\operatorname{EGARCH}(2,2,2)$ & Normal & 0,034947 & 1,975929 & $-6,322589$ & $-6,304279$ \\
\hline $\operatorname{EGARCH}(2,2,2)$ & T-student & 0,033118 & 1,928357 & $-6,429970$ & $-6,409830$ \\
\hline $\operatorname{EGARCH}(2,2,1)$ & Normal & 0,034870 & 1,966502 & $-6,321266$ & $-6,304787$ \\
\hline $\operatorname{EGARCH}(2,2,1)$ & T-student & 0,033093 & 1,929906 & $-6,429214$ & $-6,410905$ \\
\hline $\operatorname{EGARCH}(2,1,2)$ & Normal & 0,034978 & 1,968930 & $-6,321805$ & $-6,305327$ \\
\hline $\operatorname{EGARCH}(2,1,2)$ & T-student & 0,033082 & 1,927538 & $-6,430057$ & $-6,411748$ \\
\hline $\operatorname{EGARCH}(1,2,2)$ & Normal & 0,034785 & 1,970513 & $-6,321707$ & $-6,305229$ \\
\hline $\operatorname{EGARCH}(1,2,2)$ & T-student & 0,033094 & 1,928295 & $-6,430532$ & $-6,412223$ \\
\hline $\operatorname{EGARCH}(3,1,1)$ & T-student & 0,033161 & 1,930547 & $-6,428862$ & $-6,410552$ \\
\hline $\operatorname{EGARCH}(1,3,1)$ & T-student & 0,032956 & 1,929601 & $-6,428280$ & $-6,409971$ \\
\hline $\operatorname{EGARCH}(1,1,3)$ & T-student & 0,032886 & 1,926740 & $-6,429439$ & $-6,411130$ \\
\hline $\operatorname{EGARCH}(3,3,3)$ & T-student & 0,033327 & 1,935544 & $-6,430248$ & $-6,404616$ \\
\hline $\operatorname{EGARCH}(3,3,1)$ & T-student & 0,033148 & 1,929981 & $-6,428018$ & $-6,406047$ \\
\hline $\operatorname{EGARCH}(3,1,3)$ & T-student & 0,033107 & 1,927678 & $-6,429281$ & $-6,407310$ \\
\hline $\operatorname{EGARCH}(1,3,3)$ & T-student & 0,033112 & 1,928758 & $-6,429509$ & $-6,407538$ \\
\hline $\operatorname{EGARCH}(3,21)$ & T-student & 0,033111 & 1,930307 & $-6,427896$ & $-6,407756$ \\
\hline $\operatorname{EGARCH}(3,1,2)$ & T-student & 0,033112 & 1,927772 & $-6,429878$ & $-6,409738$ \\
\hline $\operatorname{EGARCH}(1,2,3)$ & T-student & 0,033116 & 1,928760 & $-6,430106$ & $-6,409966$ \\
\hline
\end{tabular}




\begin{tabular}{|l|l|l|l|l|l|}
\hline EGARCH $(1,3,2)$ & T-student & 0,033073 & 1,928279 & $-6,429972$ & $-6,409831$ \\
\hline EGARCH $(2,3,1)$ & T-student & 0,033148 & 1,930928 & $-6,428680$ & $-6,408540$ \\
\hline EGARCH $(2,1,3)$ & T-student & 0,033952 & 1,935426 & $-6,419562$ & $-6,399422$ \\
\hline EGARCH $(3,2,2)$ & T-student & 0,033117 & 1,928778 & $-6,429483$ & $-6,407512$ \\
\hline EGARCH $(2,3,2)$ & T-student & 0,033135 & 1,928890 & $-6,429565$ & $-6,407594$ \\
\hline EGARCH $(2,3,3)$ & T-student & 0,033130 & 1,928844 & $-6,428966$ & $-6,405164$ \\
\hline GJR $(1,1,1)$ & Normal & 0,034851 & 1,965154 & $-6,314916$ & $-6,302100$ \\
\hline GJR $(1,1,1)$ & T-student & 0,033162 & 1,927181 & $-6,424715$ & $-6,410067$ \\
\hline GJR $(1,1,2)$ & T-student & 0,033148 & 1,925378 & $-6,424639$ & $-6,408161$ \\
\hline GJR $(1,2,1)$ & T-student & 0,033064 & 1,927419 & $-6,425576$ & $-6,409098$ \\
\hline GJR $(2,1,1)$ & T-student & 0,033160 & 1,927572 & $-6,424149$ & $-6,407670$ \\
\hline GJR $(2,2,2)$ & T-student & 0,033094 & 1,926311 & $-6,424810$ & $-6,404670$ \\
\hline GJR $(1,2,2)$ & T-student & 0,033099 & 1,926805 & $-6,425367$ & $-6,407058$ \\
\hline
\end{tabular}

